\title{
ELECTROCATALYTIC HYDROGENATION PROCESSES AT CONTROLLED POTENTIAL - 2. CHARGE TRANSFER AT A SLURRY ELECTRODE
}

\author{
J. F. van der Plas, E. Barendrecht and H. Zeilmaker \\ Laboralory for Electrochemistry, University of Technology, \\ P.O. Box 513, Eindhoven, The Netherlands
}

(Received 30 April 1979)

\begin{abstract}
A theoretical approach has been made to charge transfer processes in a slurry cell with catalyst particles. The processes are described with an equivalent electric circuit. Calculations have been made of the potential of the catalyst as a function of the rate of the different charge transfer processes.

Analogous calculations have been made to determine the conditions to measure the catalyst potential accurately with a measuring probe.
\end{abstract}

\section{INTRODUCTION}

The slurry electrode consists of a suspension of electron conducting catalyst particles having intermittent contacts with a feeder (collector) electrode. The system can be used to obtain high volume currents at fairly low overpotentials or to let a reaction take place at controlled potential. In the first case, the suspension can be considered as an extension of the feeder electrode. In the second case, the feeder electrode serves to impose a potential on the catalyst particles. Electrochemical reactions at the feeder electrode must be avoided, because these will not only increase the energy consumption of the potential controlling electrode, but can also appear to be unwanted side reactions.

The slurry electrode has been used to increase the current for the oxidation of hydrogen $[1-3]$ and for the hydrogen evolution reaction [4]. Others have investigated the hydrogenation of organic molecules in a slurry reactor [5].

However, the slurry electrode operating at a controlled catalyst potential has as yet been studied partly. Kinza[6, 7] has tried to control the catalyst potential via the concentration of the reactants, but the use of a feeder electrode to control the catalyst potential has as yet not been described.

It is our aim to study the conditions for controlling the catalysts potential with a feeder electrode; moreover, we present some tentative theoretical considerations on charge transfer processes in a slurry electrode. Because charge transfer processes involved in controlling the catalyst potential and in measuring this potential with a probe electrode are much alike, these problems will be reduced to the same denominator. Separately, the performance of the measuring probe will be described (part 4).

\section{THEORETICAL CONSIDERATIONS}

\subsection{Charge transfer processes at a particle}

The rate of an electrochemical reaction is linearly dependent on the charge transfer exchange between the catalyst system and the reactants (Fig. 1). And because the potential of the eatalyst system controls this rate, control of the catalyst potential seems to be of utmost importance. The charge transferred between feeder electrode and particle (Fig. 1b) must then only be used to maintain the potential of the particles on its optimal value at which the net charge transfer exchange is at a minimum. Apart from the two mentioned charge transfer processes, charge is also transferred from one particle to another (Fig. 1d) and between the feeder electrode and the reactants (Fig. 1c). The undesired electrochemical reaction at the feeder electrode can be largely avoided by proper choice of the material of the feeder electrode. Charge transfer between two or more particles will be small due to the fact, that on the average, the particles will have the same potential. A simplified equivalent circuit can be designed which describes these two processes (Fig. 2). This circuit deseribes the particle as a capacitance with a value $C_{p}$ at a potential $V_{p}$, which is (dis)charged, via an electrochemical reaction resistance, $R_{E}$, by means of a net charge transfer exchange with the reactants, described as a bulk capacitance $C$ at the redoxpotential $V$, and (dis)charged via a contact resistance, $R_{c}$, by the feeder electrode at potential $E$.

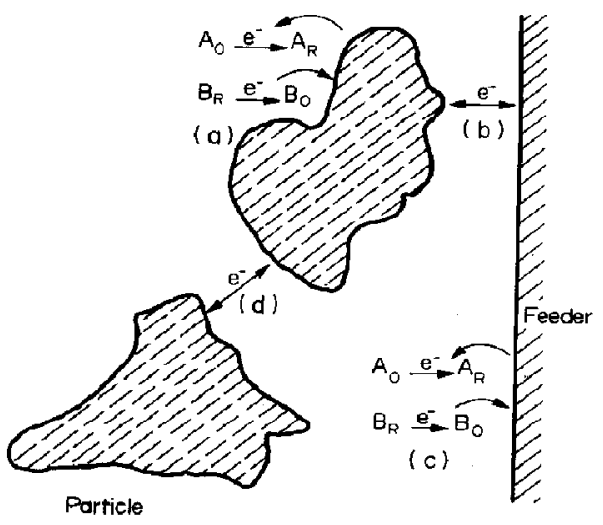

Fig. 1. Scheme of possible charge transfer processes. 


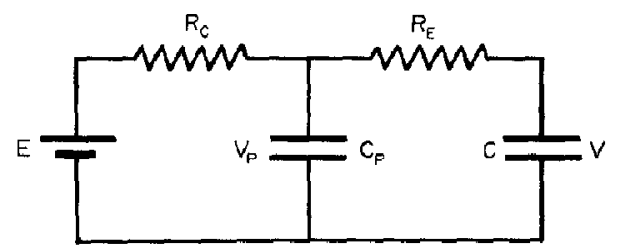

Fig. 2. Equivalent electrical circuit for the charge transfer between a feeder electrode and a particle.

The potential of the particle, $V_{p}$, can be calculated from this circuit as a function of the time elapsed since the particle and feeder electrode made contact. Solving the following four equations with four unknowns (see Fig. 2).

$$
\begin{aligned}
i_{p} & =C_{p} \frac{\partial V_{p}}{\partial t} \\
\left(i-i_{p}\right) & =C \frac{\partial V}{\partial t} \\
E & =V_{p}+i R_{c} \\
V_{p} & =V+\left(i-i_{p}\right) R_{E}
\end{aligned}
$$

leads to a second order differential equation:

$$
\begin{aligned}
\frac{\partial^{2} V_{p}}{\partial t^{2}}+\left(\frac{1}{R_{c} C_{p}}+\frac{1}{C R_{E}}+\right. & \left.\frac{1}{C_{p} R_{E}}\right) \\
& \times \frac{\partial V_{p}}{\partial t}-\frac{\left(E-V_{p}\right)}{C C_{p} R_{c} R_{E}}=0
\end{aligned}
$$

This second order differential equation can be solved, using the following boundary conditions.

$$
t=0 ; \quad V=V_{p}=V_{p}(0)
$$

The solution of the differential equation reads after making it dimensionless with:

$$
\begin{aligned}
& R_{c} / R_{E}=\alpha ; \quad C / C_{p}=\beta ; \\
& t / 2 R_{c} C_{p}=X ; \quad \frac{V_{p}-E}{\left(V_{p}\right)_{t=0}-E}=Y \\
& Y=A_{+} \exp \left(D_{+} X\right)+A_{-} \exp \left(D_{-} X\right)
\end{aligned}
$$

with

$$
\begin{aligned}
& D_{ \pm}=(-1 / \beta)[(\alpha+\alpha \beta+\beta) \pm \\
&\left.\left\{(\alpha+\alpha \beta+\beta)^{2}-4 \alpha \beta\right\}^{1 / 2}\right] \\
& A_{ \pm}=\frac{1}{2}\left[\begin{array}{c}
\left\{(\alpha+\alpha \beta+\beta)^{2}-4 \alpha \beta\right\}^{1 / 2} \\
\frac{ \pm(\beta-\alpha \beta-\alpha)}{\left\{(\alpha+\alpha \beta+\beta)^{2}-4 \alpha \beta\right\}^{1 / 2}}
\end{array}\right]
\end{aligned}
$$

This equation is plotted as a function of $\alpha=R_{c} / R_{E}$ (Fig. 3) and of $\beta=C / C_{p}$ (Fig. 4) to illustrate the effect of $\alpha$ and $\beta$ on the time necessary to equalize the catalyst potential and the feeder potential. From Fig. 3 the following cases are evident:

If $R_{c} \ll R_{E}(\alpha \ll 1)$, the relaxation time of the catalyst potential depends solely on the value of $\boldsymbol{R}_{c}$ and $C_{p}$. The charge transfer between the feeder electrode and the particle is much faster than the charge transfer between the particle and the reactants. Therefore the

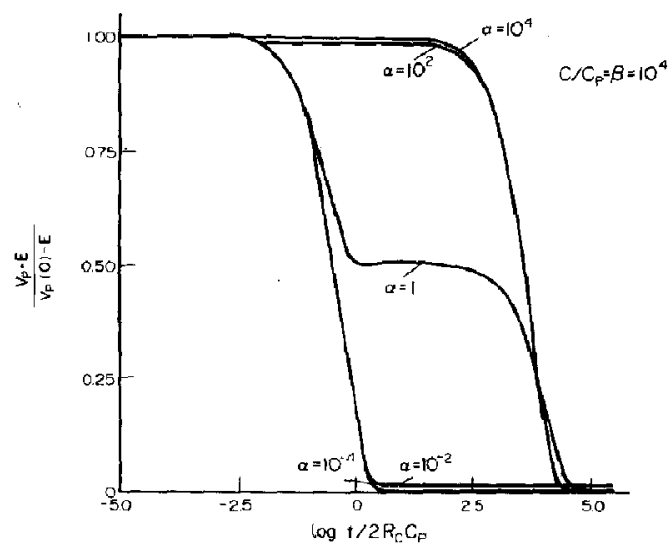

Fig. 3. Plot of equation (3) for different values of $\alpha . \beta=10^{4}$.

time necessary to change the catalyst potential to the feeder electrode potential is much smaller than the time needed to change the particle potential back to its initial value by means of the net electrochemical reactions. In this situation, control of the catalyst potential is possible provided the contact time between feeder electrode and particle is in the order of the relaxation time or less.

- If $R_{c} \approx R_{E}(\alpha \approx 1)$, the potential of the particle varies slowly in time compared to the above mentioned situation. In this case, part of the charge transferred between the feeder and the particle is consumed by an electrochemical reaction. This leads to a particle potential between the feeder potential and the redoxpotential of the solution. In the long run the reactants in the bulk will have reacted and then the particle will change further towards the feeder potential. The particle potential can thus be influenced although the relaxation time is not as fast as in the previous case.

- When $R_{c} \gg R_{E}(\alpha \gg 1)$, the particle potential is not influenced at all by contact with the feeder electrode. Only when the substrates present have reacted, the potential of the particle will be influenced by the

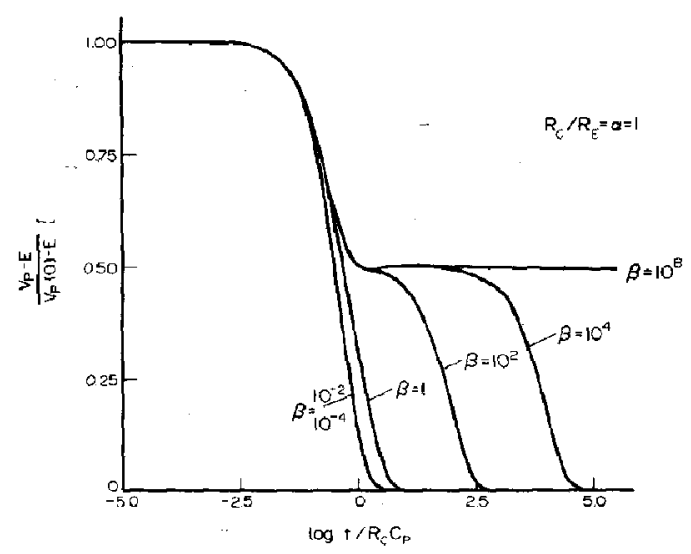

Fig. 4. Plot of equation (3) for different values of $\beta . \alpha=1$. 
feeder electrode. In this situation, however, no reaction takes place anymore, so that control of the catalyst potential during the reaction is not possible.

From the above mentioned cases, it can be concluded that, to control the catalyst potential with a feeder electrode, the electrochemical reaction resistance $R_{E}$ should be smaller than the contact resistance of particle with feeder electrode, $R_{c}$. Furthermore, the contact time of particle with the feeder electrode should be in the order of the relaxation time $\left(R_{c} C_{p}\right)$ of the particle in order to realize a rapid change of the catalyst potential.

\subsection{Charge transfer processes at a measuring probe}

The charge transfer processes between a catalyst and a measuring probe, leading to the actual catalyst potential, are very much alike the charge transfer processes described in the previous section. In this case, also an equivalent circuit can be made, which evaluates the influence of the various parameters (Fig. 5). In this circuit the particle, respectively the measur. ing probe, are described as capacities $C_{p}$, respectively $C_{m}$, at potentials $V_{p}$, respectively $\dot{V}_{m}$, in contact with each other (contact resistance $R_{2}$ )

Both capacities are also influenced by electrochemical reactions that take place at the surface of particles and the measuring probe. These reactions are represented by voltage sources with a potential equal to the redoxpotential of the reactants at the surfaces of the catalyst $\left(V_{p}(0)\right)$ and the measuring probe $\left(V_{m}(0)\right)$. The electrochemical reaction resistances $R_{1}$ and $R_{3}$. The potential-time relationship of the measuring probe can be calculated from this circuit in the same manner as in the previous section, using the following boundary conditions:

$$
\begin{aligned}
t=0 & V_{m}=V_{m}(0) \\
V_{p} & =V_{p}(0)
\end{aligned}
$$

The potential of the measuring probe is then given by:

$$
\begin{aligned}
Y=A_{+} \exp \left(D_{+} X\right)+A_{-} \exp \left(D_{-} X\right) & \\
& +\frac{\alpha \beta}{(\alpha+\beta+\alpha \beta)}
\end{aligned}
$$

with

$$
\begin{aligned}
D_{ \pm}= & -\left[\frac{(1+\alpha+\alpha \gamma+\beta \gamma)}{\gamma}\right. \\
& \pm\left\{\left(\frac{1+\alpha+\alpha \gamma+\beta \gamma}{\gamma}\right)^{2}\right. \\
& \left.\left.-4\left(\frac{\alpha+\alpha \beta+\beta}{\gamma}\right)\right\}^{1 / 2}\right]
\end{aligned}
$$

This function has been plotted to evaluate the potential response of the measuring probe as a function of the variables used. Figure 6 shows the dependence of the potential of the measuring probe as a function of $\alpha$. The rise of the potential of the measuring probe, after contact is made between catalyst and measuring probe, depends on the value of the contact resistance, $R_{2}$. After the potential of the measuring probe has reached a maximum value, it drops again due to the discharge of capacities, $C_{m}$ and $C_{p}$, by potential source $V_{m}(0)$. The maximum potential drop is a function of $\beta$ as is shown in Fig. 7. When $\beta \gg 1$, no potential drop occurs at all and the initial maximum value, which depends on $\alpha$ (see Fig. 6), is maintained. The important conclusion, that thus can be reached, is that the net electrochemical reaction at the surface of the measuring probe should be small compared to the net reactions at the particle surface. Furthermore, the contact resistance should be small compared to the electrochemical reaction at the surface of the measuring probe. When these two conditions are fulfilled, the ratio of $R_{2}$ and $R_{3}$ is of no importance to the potential response of the measuring probe.

The influence of $\gamma$ on the potential response is given in Fig. 8, which shows that a small value of $\gamma$ (ie, a small capacity of the measuring probe compared to the capacity of the particles) gives a faster potential change of the measuring probe. This means that small measuring probes should be used to measure a catalyst potential.

The discrepancy between the potential of the measuring probe and the catalyst particle is of course a function of the difference in redoxpotential of the reactants at the surfaces of the measuring probe and the catalyst particles $\left(V_{n}(0)-V_{\nu}(0)\right)$. When both measuring probe and catalysts are of the same material, these potentials will not differ much and an accurate measurement of the catalyst potential is possible. However, when the potential of the catalyst is controlled by means of a feeder electrode, the voltage $V_{p}(0)$ is not due to the net electrochemical reactions at the particle surface, but equal to the feeder electrode potential. The electrochemical reaction resistance is then replaced by the contact resistance $R_{c}$ between the feeder electrode and the particles. In this situation, the charge transfer due to the electrochemical reaction rate at the surface of the measuring probe, should be small compared to the charge transfer between the feeder electrode and the particles.

\section{CONCLUSION}

From the equivalent circuits used to describe charge transfer processes between a feeder electrode or a

$$
\left.A_{ \pm}= \pm\left[\frac{\left(\alpha^{2} \beta \gamma+\alpha \beta^{2} \gamma-\alpha^{2} \beta-\alpha \beta-2 \alpha^{2}\right)}{2 \gamma(\alpha+\beta+\alpha \beta)\left\{\left(\frac{1+\alpha+\alpha \gamma+\beta \gamma}{\gamma}\right)^{2}-4\left(\frac{\alpha+\beta+\alpha \beta}{\gamma}\right)\right\}^{1 / 2}}\right]\right]
$$

$$
-\frac{\alpha \beta}{2(\alpha+\alpha \beta+\beta)} \text {. }
$$

$$
\begin{aligned}
& \alpha=R_{1} / R_{2} ; \quad \beta=R_{1} / R_{3} ; \gamma=C_{m} / C_{p} ; \\
& X=t / 2 R_{1} C_{p} ; \quad Y=\frac{V_{m}-V_{m}(0)}{V_{p}(0)-V_{m}(0)} .
\end{aligned}
$$

measuring probe and the catalyst particles, the conditions can be evaluated prescribing the effectiveness of potential control and catalyst potential measurement. With knowledge of the actual values of the capacities and resistances in a given system the 


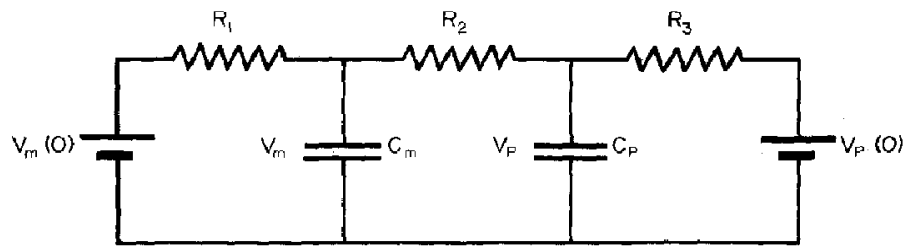

Fig. 5. Equivalent electrical circuit for the charge transfer between a measuring probe and a particle.

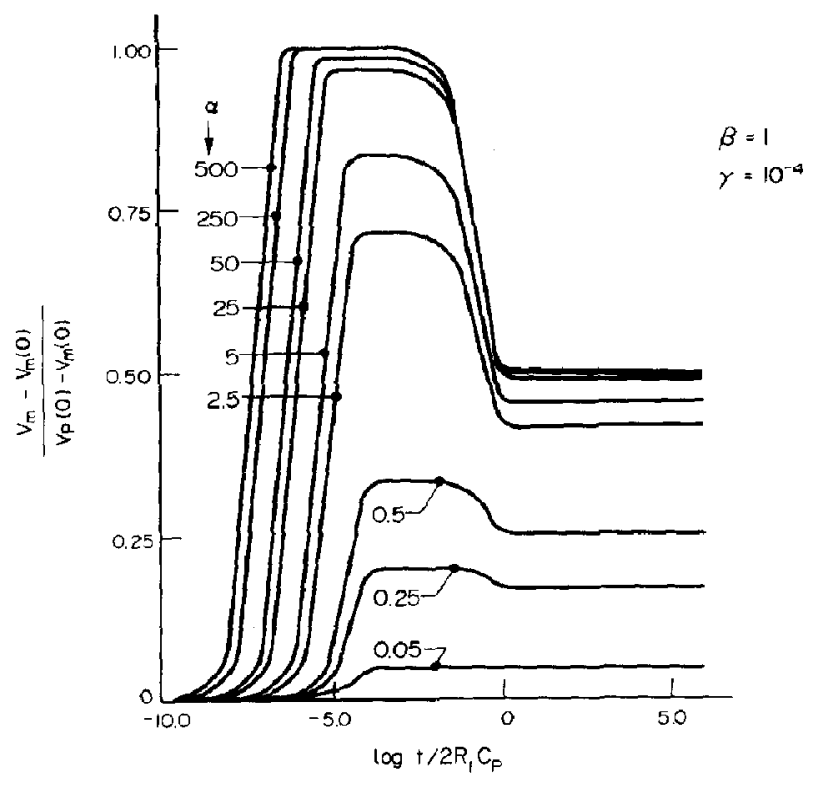

Fig. 6. Plot of equation (6) for different values of $\alpha . \beta=1 ; \gamma=10^{-4}$.

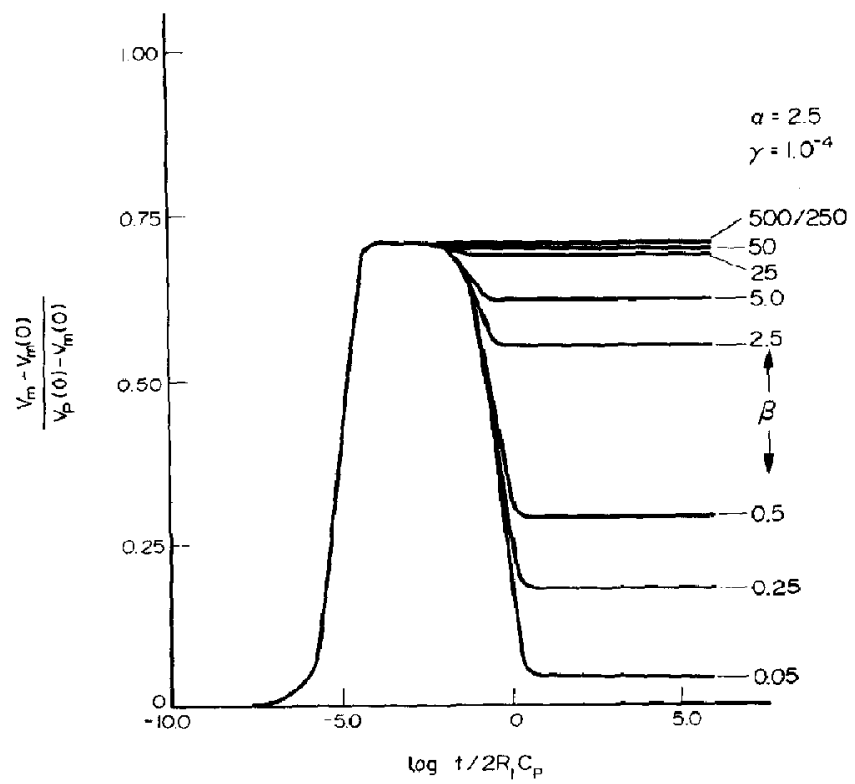

Fig. 7. Plot of equation (6) for different values of $\beta . \alpha=2.5 ; \gamma=10^{-4}$. 


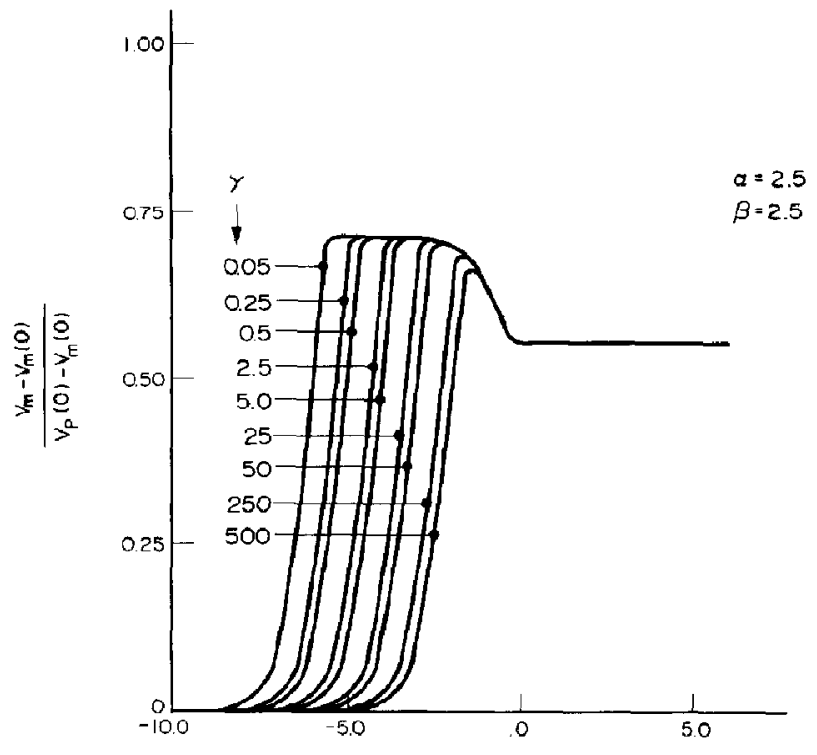

$\log t / 2 R_{2} C_{p}$

Fig. 8. Plot of equation (6) for different values of $\gamma \cdot \alpha=2.5 ; \beta=2.5$.

possibilities of controlling and/or measuring the catalyst potential can bc predicted. Although the models used are only a first approximation to the real situation it gives a sound basis on which further experimental work can be done.

Acknowledgement - The authors thank Prof. M. Tels for valuable discussions. This work has been carried out with financial support from the Netherlands Organization for the Advancement of Pure Research (ZWO).

\section{REFERENCES}

1. H. Gerischer, Ber. Bunsinges. Phys. Chemie 67, 164-167 (1963).

2. J. Held and H. Gerischer, Ber. Bunsenges. Phys. Chemie 67, 921-929.

3. E. Keren and A. Soffer, J. electroanal. Chem. 44, 53-62 (1973).

4. R. M. Lazorenko-Manevich and A. V. Ushakov, Dokl. Akad. Nauk. SSSR 161, 156-159 (1965).

5. D. V. Sokolskii, Progress in Echem. of Organic Compounds Vol. 1, (Edited by A. N. Frumkin and A. B. Ershler) Phenum Press, London New York (1971).

6. H. Kinza, Z, Phys. Chemie, Leipzig 255, 180-192 (1974).

7. H. Kinza, Z. Phys. Chemie, Leipzig 255, 517-537 (1974). 